\title{
Design of a New Radio-Frequency Switch Topology with Very Low Polarization Current
}

\author{
Fethi MEJRI, Taoufik AGUILI
}

\author{
Sys'Com Research Laboratory, National Engineering School of Tunis (ENIT), \\ University Tunis El Manar, Tunis, Tunisia
}

fethi_mejri@laposte.net, taoufik.aguili@gmail.com

Submitted November 8, 2018 / Accepted May 1, 2019

\begin{abstract}
Embedded systems are generally powered by batteries, characterized by a limited duration of electrical energy storage. For that, low power consuming RF components are often used. This work focuses on the design of a new RF switch based on one varicap diode within a series/parallel circuit topology. The latter is low-power-consuming in both ON and OFF mode and inhibits eventual nonlinearities caused by series diodes such as common PIN diodes. Before performing simulations, the diode has been characterized experimentally in the whole UHF band of interest and the extracted S-parameters have been used in the simulations of the resonating structure. The optimized switch has been measured and has showed a good agreement with simulated results in terms of high isolation $(50 \mathrm{~dB})$ and low insertion loss $(0.1 \mathrm{~dB})$. These results showed better performances compared to other structures based on RF MEMS, PIN diode or FET transistor devices, available in the literature.
\end{abstract}

\section{Keywords}

RF switch, microstrip structure, microwave, varicap diode, parallel and series resonance, reconfigurable resonant circuit, very low polarization current

\section{Introduction}

The RF switches commonly used in telecommunication systems are based essentially on PIN diodes (Positive Intrinsic Negative diode) [1], [2], FET transistors (Field Effect Transistor) [3], [4] and on RF MEMS (Micro Electro Mechanical Systems) [5], [6]. These RF switches have advantages and disadvantages. A PIN diode RF switch can handle considerable power and produce less distortion, to the detriment to a switching time that is longer and that requires a much greater control current. A FET transistor switch can operate with a very low polarization current, making it very well suited for battery powered applications. The latter can also be incorporated into integrated circuits. An RF MEMS switch provides a much lower switching speed than the FET transistors. It has a very low insertion loss due to the low resistivity of the metal, high isolation due to the physical separation of the contacts and good linearity with respect to PIN diodes and FET transistors.

Developers of radio frequency devices powered by a battery require RF circuits that consume as little power as possible. This has encouraged us to contribute to the development of new RF components that require low power consumption.

Table 1 presents the characteristics of our RF switch and of some RF (PIN diode, FET transistor and RF MEMS) switches commonly used in telecommunication systems [7].

In this article, we designed, realized and characterized a new RF switch topology. Its operation requires a very low consumption of electrical energy. It is based on a resonant circuit, which is placed in parallel with the load (Fig. 1(A), (B) and (C)). The resonant circuit is controlled by a varicap diode [8]. The DC voltage command of this diode switches the resonant circuit of a series resonance (open switch) to a parallel resonance (closed switch). This switch has multiple advantages such as low manufacturing cost, simplicity of design and ease of reproducibility. It can be used in telecommunication applications with a narrow frequency band or at a single-frequency, such as reconfigurable antennas [9], [10].

The varicap diode, generally used as a capacitor, is used here to achieve series and parallel resonance yielding hence an open or short circuit. Among its advantages are the low energy consumption, low cost, and scalability due to large tuning possibilities.

\begin{tabular}{|l|c|c|c|c|}
\hline \multicolumn{1}{|c|}{ Parameters } & MESFET & PIN diode & MEMS & Our Work \\
\hline Series resistance $(\Omega)$ & 3 to 5 & 1 & $<1$ & $<1$ \\
\hline Loss $(\mathrm{dB})$ & 0.5 to 1.0 & 0.5 to 1.0 & 0.1 & 0.1 \\
\hline Isolation $(\mathrm{dB})$ & 20 to 40 & 40 & $>40$ & $>40$ \\
\hline Switching speed & $\sim \mathrm{ns}$ & $\sim \mu \mathrm{s}$ & $\sim \mu \mathrm{s}$ & $\sim \mu \mathrm{s}$ \\
\hline Control voltage $(\mathrm{V})$ & 8 & 3 to 5 & 3 to 30 & 6.5 to 12.2 \\
\hline Control current & $<10 \mu \mathrm{A}$ & $10 \mathrm{~mA}$ & $<10 \mu \mathrm{A}$ & $<10 \mathrm{nA}$ \\
\hline
\end{tabular}

Tab. 1. Comparison between our RF switch and some RF switches commonly used. 


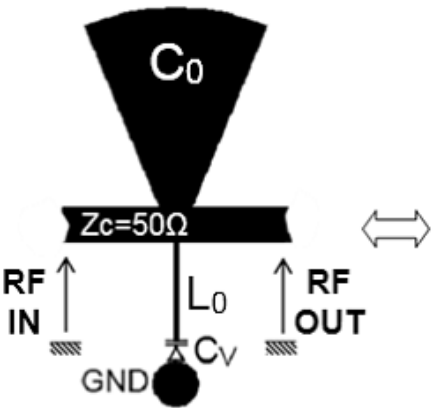

(A)

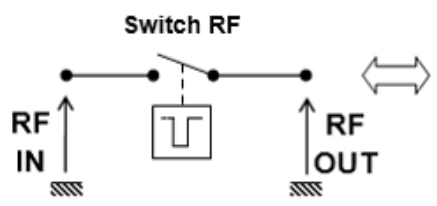

(D)

\section{$\mathrm{C}_{0}=7 \mathrm{pF}$ \\ $\mathrm{L}_{0}=5 \mathrm{nH}$}

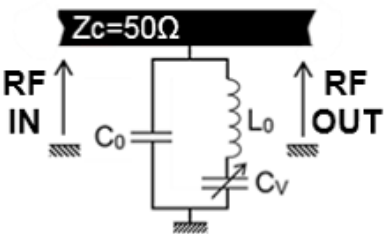

(B)

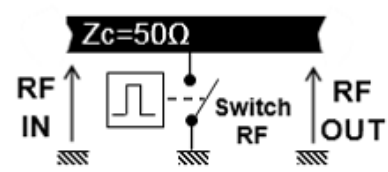

(C)

Fig. 1. (A): Realized resonant circuit in microstrip technology. (B): Equivalent electrical diagram of the studied resonant circuit. (C), (D): Synoptic diagram of the studied RF switch.

\section{Characterization of the RF Switch}

The RF switch was made using microstrip technology [11] and tested at $925 \mathrm{MHz}$. It is implemented on a circuit board that is frequently available and inexpensive. The substrate is Teflon glass of relative permittivity $\varepsilon_{\mathrm{r}}=4.32$, of thickness $H=1.5 \mathrm{~mm}$ and having losses modeled by $\tan \delta=0.02$. The conductor is the copper of conductivity $\sigma=59.6 \times 10^{6} \mathrm{~S} / \mathrm{m}$ and thickness $T=35 \mu \mathrm{m}$.

\subsection{Description of the Realized RF Switch}

Figures 1(C), (D) show the block diagrams of the realized RF switch.

Figure 2 shows the RF switch made. It consists of a bias tee, providing isolation between the RF signal and the source delivering the control DC voltage, allowing the variation of the capacitance of the varicap diode. A resonant circuit placed in parallel with the load (Fig. 1(A), (B)

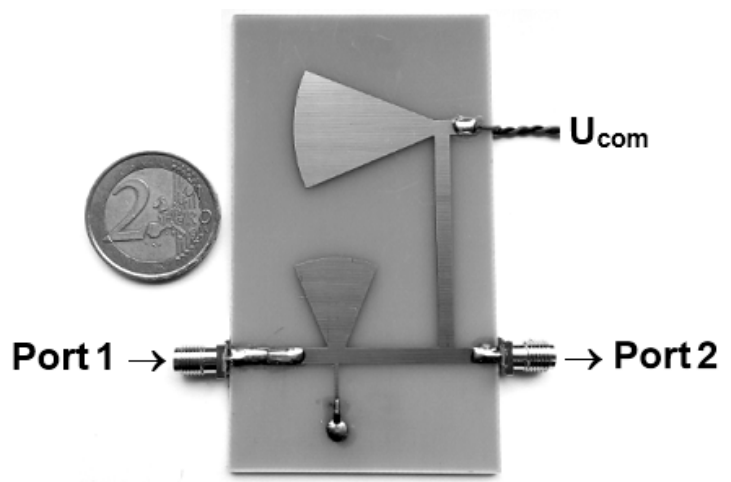

Fig. 2. Topology and design of the RF switch.

and Fig. 2). A microstrip line, with characteristic impedance $Z_{\mathrm{C}}=50 \Omega$, is placed between the input/output ports of the RF switch. The latter has a width of the track equal to $2.86 \mathrm{~mm}$, calculated by the LineCalc software of ADS (Advanced Design System).

\section{- Polarization tee}

The polarization of the varicap diode is through a bias tee (isolation circuit) shown in Fig. 3. This bias tee is a passive component, made in microstrip technology. It is a low-pass filter, which passes the direct current of the control source to the varicap diode and blocks the passage of the RF signal to the control source, so that it is not disturbed. The latter consists of distributed elements, a line of length $L=\lambda_{\mathrm{g}} / 4=44.836 \mathrm{~mm}$ and characteristic impedance $Z_{\mathrm{C}}=50 \Omega(W=2.86 \mathrm{~mm}$ : width of the track corresponding to $Z_{\mathrm{C}}=50 \Omega$ ), followed by a planar capacitor, placed in parallel with the control source. This capacitor has the shape of a butterfly, radius $R=27 \mathrm{~mm}$ and angle $\theta=45^{\circ}$. These elements make it possible to inject a continuous current (or a voltage) into the RF circuit without altering the RF signal in the transmission chain. The shape of the butterfly capacitor [12], [13] allows it to operate in a certain broad band. It also reduces its radiation with respect to a capacitor of square or rectangular shape. Its length is chosen equal to $\lambda_{\mathrm{g}} / 4$. It is obtained only by an optimization under the ADS software. And this since its width is increasing. So its characteristic impedance $\left(Z_{\mathrm{C}}\right)$ and its effective permittivity $\left(\varepsilon_{\text {eff }}\right)$ vary according to its length.

$$
L=\frac{\lambda_{g}}{4}=\frac{c}{4 f \cdot \sqrt{\varepsilon_{\text {eff }}}}
$$

with $c=3 \times 10^{8} \mathrm{~m} / \mathrm{s}$, speed of light in vacuum.

The butterfly capacitor is a planar line [13] which obeys the relation (2)

$$
Z(x)=Z_{\mathrm{C}} \frac{Z_{\mathrm{L}}+\mathrm{j} Z_{\mathrm{C}} \tan (\beta x)}{Z_{\mathrm{C}}+\mathrm{j} Z_{\mathrm{L}} \tan (\beta x)}
$$

where $Z_{\mathrm{L}}$ represents the impedance of the load and $\beta$ represents the propagation constant.

The length of this line is equal to $\lambda_{\mathrm{g}} / 4$ and it's open at its end $\left(Z_{\mathrm{L}}=\infty\right)$. It brings back to its input a short circuit for the RF signal

$$
Z\left(x=\frac{\lambda}{4}\right)=0 .
$$

The short circuit brought back to the capacitor input is located at the exit of a line of length $\lambda_{\mathrm{g}} / 4$. This line brings back to its input an open circuit for the RF signal

$$
Z\left(x=\frac{\lambda}{4}\right)=\infty .
$$

Figure 3 shows the parameters $S$, of the polarization tee, obtained by simulations under the ADS software and by measurements using a vector network analyzer (VNA). Both results are in good agreement. 


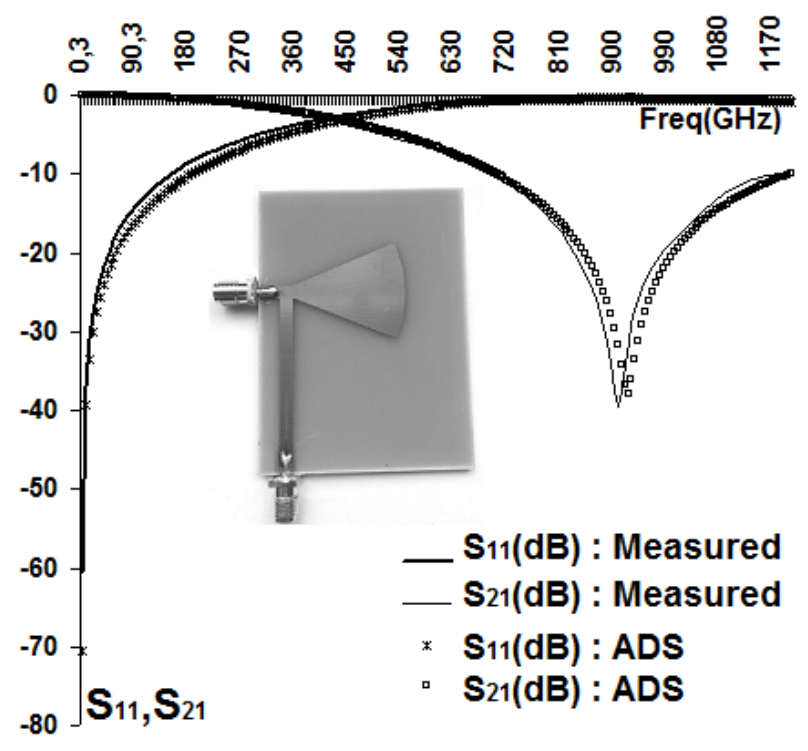

Fig. 3. $S_{i j}$ parameters of the polarization tee.

Figure 3 clearly shows that for a direct current the reflection coefficients $\left(S_{11} \approx S_{22}\right)$ are negligible, of the order of $-70 \mathrm{~dB}$, and the transmission coefficients $\left(S_{21} \approx S_{12}\right)$ are maximum, of the order of $0 \mathrm{~dB}$. On the other hand, for the RF signal at $925 \mathrm{MHz}$, the reflection coefficients are maximum, equal to almost $0 \mathrm{~dB}$, and the transmission coefficients are negligible, equal to $-31 \mathrm{~dB}$. We also note from Fig. 3 that the bandwidth of this isolation circuit is of the order of $500 \mathrm{MHz}$. On this frequency band, the reflection coefficients are strictly greater than $-3 \mathrm{~dB}$ and the transmission coefficients are strictly less than $-10 \mathrm{~dB}$. We can also say that the bandwidth of the RF switch is related to this isolation circuit. In conclusion, the realized isolation circuit makes it possible to block the RF signal and lets the control DC voltage towards the varicap diode.

\section{- Resonant series/parallel circuit}

Figure 4 shows the series/parallel resonant circuit studied. It is composed of an inductance $L_{0}$ placed in series with a varicap diode, variable capacitance $C_{\mathrm{v}}$ and resistor series $r_{\mathrm{S}}$ assumed negligible. These two elements are placed in parallel with a capacitor $C_{0}$. The inductance $L_{0}$ and the capacitor $C_{0}$ are produced using microstrip technology (Fig. 1(A)), with $C_{0}=7 \mathrm{pF}, L_{0}=5 \mathrm{nH}$ and $f_{\mathrm{r}}=925 \mathrm{MHz}$.

The equivalent impedance of the resonant circuit is expressed by (5)

$$
Z=\mathrm{j}\left(\frac{1-L_{0} C_{\mathrm{V}} \omega^{2}}{L_{0} C_{0} C_{\mathrm{V}} \omega^{3}-\left(C_{0}+C_{\mathrm{V}}\right) \omega}\right) .
$$

The series resonance of the circuit is obtained when:

$$
Z=0 \quad \Rightarrow \quad C_{\mathrm{V}}=\frac{1}{L_{0} \omega^{2}}=5.9 \mathrm{pF} .
$$

The parallel resonance of the circuit (anti-resonance) is obtained when

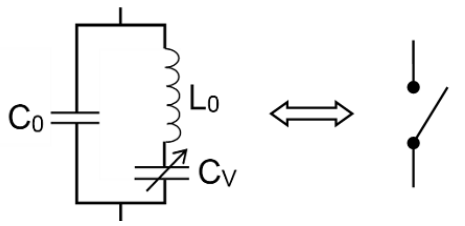

Fig. 4. Equivalent electrical diagram of the studied resonant circuit.

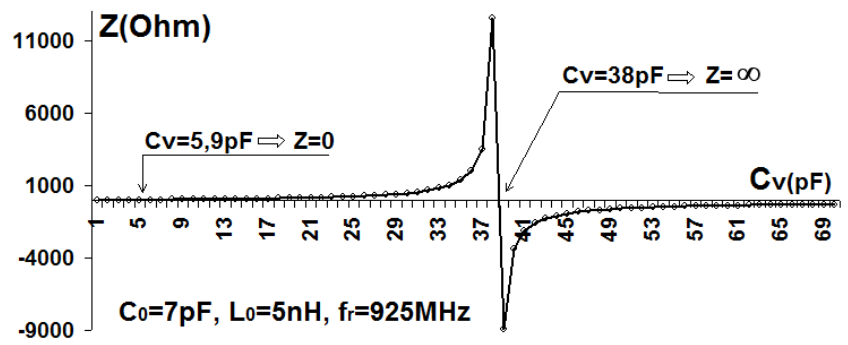

Fig. 5. Impedance of the resonant circuit as a function of the capacitance $\mathrm{C}_{\mathrm{v}}$.

$$
Z=\infty \Rightarrow C_{\mathrm{v}}=\frac{C_{0}}{L_{0} C_{0} \omega^{2}-1}=38 \mathrm{pF} .
$$

Figure 5 shows the variation of the impedance $Z$ of the circuit of Fig. 4 as a function of the capacitance $C_{\mathrm{v}}$. The resonances of this circuit, in series mode and in parallel mode, at $925 \mathrm{MHz}$, are obtained respectively for the capacitance of the varicap diode $C_{\mathrm{v}}=5.9 \mathrm{pF}$ and $C_{\mathrm{v}}=38 \mathrm{pF}$.

In this work, we have taken advantage of the behavior of the propagation lines to realize, in microstrip technology, the inductance $L_{0}$ and the capacitance $C_{0}$ (without discrete elements inductance and capacitance) [13]. We know that a transmission line, with a characteristic impedance $Z_{\mathrm{C}}$, closed on an impedance load $Z_{\mathrm{L}}$, has at a distance $x$ from the latter an impedance $Z(x)$ given by (2). For a transmission line whose length $x<\lambda_{\mathrm{g}} / 12$, the $\tan (\beta x)$ approximates $10 \%$ close to the product $\beta x$. In these conditions we can write:

$$
Z(x)=Z_{\mathrm{C}} \frac{Z_{\mathrm{L}}+\mathrm{j} Z_{\mathrm{C}} \beta x}{Z_{\mathrm{C}}+\mathrm{j} Z_{\mathrm{L}} \beta x} .
$$

a) For a short-circuited line $\left(Z_{\mathrm{L}}=0\right)$ or a very large characteristic impedance in front of the impedance of the load $\left(Z_{\mathrm{L}}<<Z_{\mathrm{C}} \beta x\right)$, the ratio $w / h<1$ and $\varepsilon_{\mathrm{r}}$ is low. Equation (8) then becomes:

$$
Z(x)=\mathrm{j} Z_{\mathrm{C}} \beta x=\mathrm{j} Z_{\mathrm{C}} \frac{\omega}{v_{\rho}} x=\mathrm{j} L_{0} \omega .
$$

Such a line therefore behaves as an inductive impedance $\mathrm{j} L_{0} \omega$ with:

$$
L_{0}=Z_{\mathrm{C}} \frac{x}{v_{\rho}} \text {. }
$$

b) For a line in open circuit $\left(Z_{\mathrm{L}}=\infty\right)$ or of characteristic impedance very weak in front of the load $\left(Z_{\mathrm{C}}<<Z_{\mathrm{L}} \beta L\right)$, the ratio $w / h>1$ and $\varepsilon_{\mathrm{r}}$ is high. Equation (8) then becomes: 


$$
Z(x)=-\mathrm{j} \frac{Z_{\mathrm{C}}}{\beta x}=-\mathrm{j} Z_{\mathrm{C}} \frac{v_{\rho}}{\omega x}=-\frac{\mathrm{j}}{C_{0} \omega} .
$$

The line is therefore equivalent to a value of capacity:

$$
C_{0}=\frac{x}{Z_{\mathrm{C}} v_{\rho}}
$$

The speed of propagation of the wave $v_{\rho}$ on a real microstrip line (or phase velocity) is expressed by:

$$
v_{\rho}=\frac{c}{\sqrt{\varepsilon_{\text {eff }}}}=\lambda_{g} f
$$

c) The realization, in microstrip technology, of the inductance $L_{0}$ and the capacitance $C_{0}$ follows from all that has been previously explained. Figure 5 shows the variations of the characteristic impedance $Z_{C}$ and the effective permittivity $\varepsilon_{\text {eff }}$ of a microstrip line as a function of its width $w$. They show that the characteristic impedance is inversely proportional to $w$ and that the effective permittivity of the dielectric is proportional to $w$. These results were calculated by the ADS LineCalc software, for the printed circuit defined above and for a frequency $f=$ $925 \mathrm{MHz}$.

The $L_{0}$ series inductance is obtained by a strong narrowing of the metal ribbon. A microstrip line of characteristic impedance $Z_{C}=100 \Omega$ is chosen. Based on Fig. 6, we deduce the width of its metal strip $w=0.630272 \mathrm{~mm}$ and its effective permittivity $\varepsilon_{\mathrm{eff}}=2.908$. The length of this line is calculated using (14) deduced from (10) and (13)

$$
x=\frac{c L_{0}}{Z_{\mathrm{C}} \sqrt{\varepsilon_{\text {eff }}}}=8.766 \mathrm{~mm} .
$$

A high characteristic impedance line can be identified with an $L_{0}$ series inductor and two shunt capacitances $C$. It is assumed that the influence of these abilities is negligible [13-15].

The parallel capacitance $C_{0}$ is obtained by a large expansion of the metal ribbon. A microstrip line of characteristic impedance $Z_{C}=20 \Omega$ is chosen. Based on Fig. 6, we deduce the width of its metal tape $w=10.4742 \mathrm{~mm}$ and its effective permittivity $\varepsilon_{\mathrm{eff}}=3.7$. The length of this line is calculated using (15) deduced from (12) and (13)

$$
x=\frac{c C_{0} Z_{\mathrm{C}}}{\sqrt{\varepsilon_{\mathrm{eff}}}}=21.83 \mathrm{~mm} .
$$

From the dimensions $w$ and $x$ of a capacity of rectangular shape, we can deduce the dimensions of a capacity of butterfly shape, radius $R$ and angle $\theta$.

$$
\theta=\pi / 4 \Rightarrow R=\sqrt{\frac{4 x w}{\pi}}=17.067 \mathrm{~mm}
$$

All these embodiments have discontinuities in the width $w$ of the microstrip line. In an open circuit line, the electric field lines do not stop abruptly at the end of the line

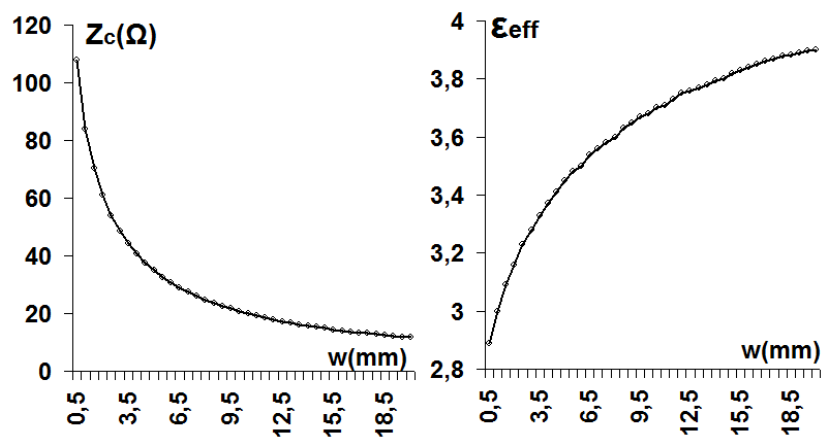

Fig. 6. Variation of $Z_{\mathrm{C}}$ and $\varepsilon_{\text {eff }}$ of a microstrip line according to its width $w(f=925 \mathrm{MHz})$.

but propagate a little further. This leads to a larger effective length [13], [15]. It is therefore necessary during the overall realization of the printed circuit to optimize, under the ADS software, the different lines of the circuit.

The varicap diode BB149A [16] was designed to work in the UHF band. For a frequency $f=1 \mathrm{MHz}$, its $C_{\mathrm{v}}$ capacity can vary from $1.951 \mathrm{pF}$ to $21.26 \mathrm{pF}$. It has a maximum series resistance $r_{\mathrm{S}}=0.75 \Omega$. These parameters change considerably for high frequencies of use. For some working frequencies and by varying the voltage $V_{\mathrm{R}}$, the varicap diode can change from a variable capacitance to a variable inductance.

The BB149A diode operates with a DC voltage and a very low reverse bias current $\left(10 \mathrm{nA}\right.$ for $V_{\mathrm{R}}=30 \mathrm{~V}$ and $T_{\mathrm{j}}=25^{\circ} \mathrm{C}$ ) [16]. This has an advantage over PIN diodes that require a much larger control current (10 mA) [7].

\subsection{Results of the Realized RF Switch}

\section{- Coefficient of reflection of the BB149A diode}

The simulation of the RF switch (Fig. 2) under the ADS software requires knowledge of the reflection coefficient (module and phase) of the varicap diode BB149A. For this, measurements of this parameter have been made using an E5062A vector network analyzer for two inverse DC voltages applied to the varicap diode (7 $\mathrm{V}$ and $11.3 \mathrm{~V})$.

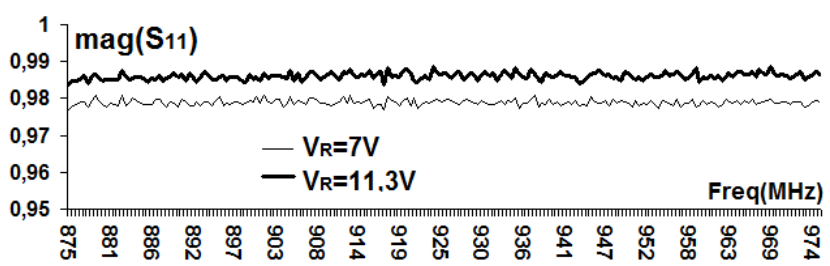

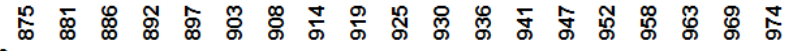

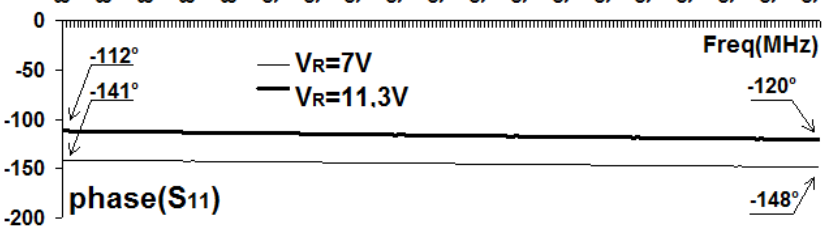

Fig. 7. Contents of the two s1p files of the BB149A varicap diode (for $V_{\mathrm{R}}=7 \mathrm{~V}$ or $V_{\mathrm{R}}=11.3 \mathrm{~V}$ ). 
These two voltages reconfigure the resonant circuit of the RF switch in parallel or series resonance mode.

Figure 7 presents the results of measurements of the reflection coefficient of the varicap diode BB149A. They are saved in Touchstone slp files [17]. They describe the actual behavior of the varicap diode BB149A when simulating the $\mathrm{RF}$ switch under the ADS software when $V_{\mathrm{R}}=7 \mathrm{~V}$ or $V_{\mathrm{R}}=11.3 \mathrm{~V}$.

\section{- Parameters S of the realized RF switch}

The application of a control inverse voltage equal to $7 \mathrm{~V}$ causes the closing of the RF switch. The resonant circuit of Fig. 2 operates in parallel resonance mode. It has an infinite impedance between the ribbon of the microstrip line and the ground plane. At this time the RF signal passes from port 1 to port 2 or vice versa (bi-directional) without any difficulty. The RF switch is transformed into a characteristic impedance transmission line $Z_{C}=50 \Omega$. This makes it possible to manage a considerable power, without distortion of the RF signal and with a low insertion loss.

Figure 8 shows a comparison between the simulation and measurement results of the RF switch (Fig. 2). They have the same variations. This figure presents the variations of the coefficients of transmission $\left(S_{21}>-1.4 \mathrm{~dB}\right)$ and of reflection $\left(S_{11}<-12.7 \mathrm{~dB}\right)$ as a function of the frequency. This for a control reverse voltage $U_{\text {com }}=V_{\mathrm{R}}=7 \mathrm{~V}$.

For a frequency $f=925 \mathrm{MHz}$, the transmission coefficient $S_{21}=-1.04 \mathrm{~dB}$ and the reflection coefficient $S_{11}=-28.3 \mathrm{~dB}$. The measurement and simulation results show the same variations and show that the RF switch is closed with a well-adapted input and output.

The application of a control reverse voltage equal to 11.3 V causes the RF switch to open. The resonant circuit of Fig. 2 operates in serial resonance mode. It has a zero impedance, which short-circuits the transmission line and blocks the passage of the RF signal from port 1 to port 2 or vice versa.

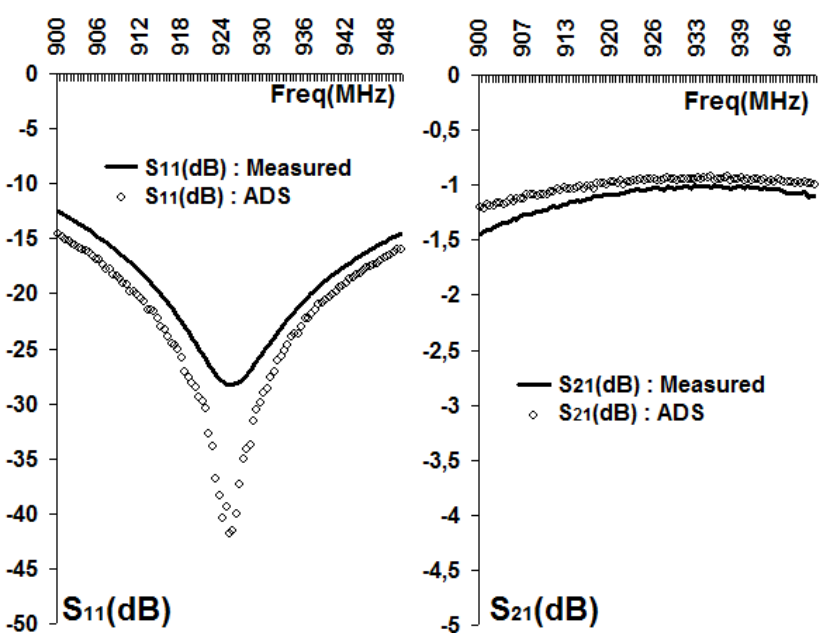

Fig. 8. Variation of $S_{11}$ and $S_{21}$ as a function of frequency $\left(V_{\mathrm{R}}=7 \mathrm{~V}\right)$

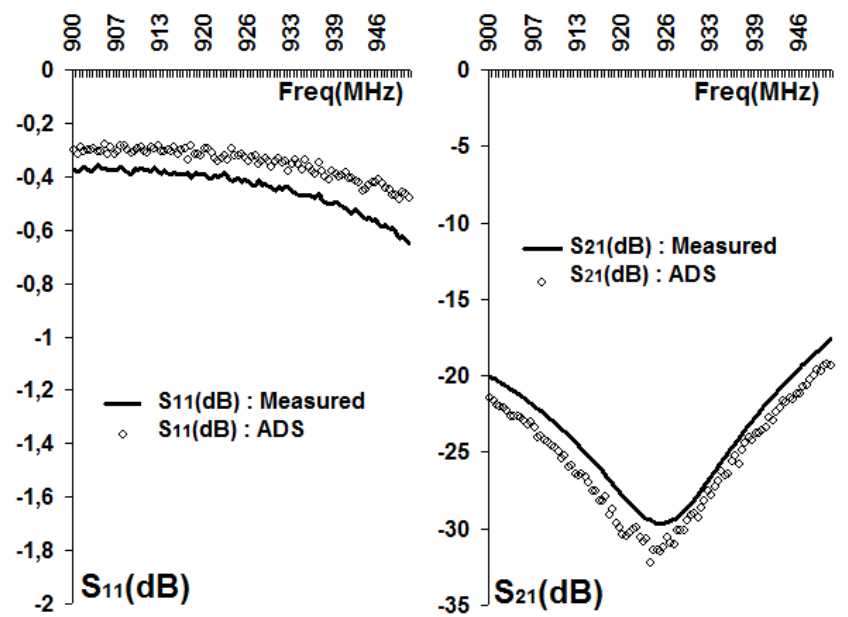

Fig. 9. Variation of $S_{11}$ and $S_{21}$ as a function of frequency $\left(V_{\mathrm{R}}=11.3 \mathrm{~V}\right)$.

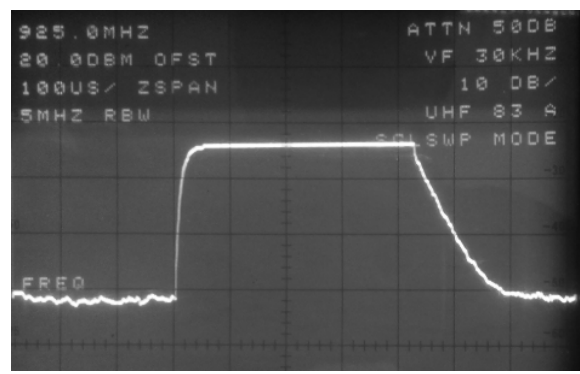

Fig. 10. Commutation time of the realized RF switch.

Figure 9 shows a comparison between the simulation and measurement results of the RF switch (Fig. 2). They are in good agreement. This figure presents the variations of the transmission coefficients $\left(S_{21}<-18 \mathrm{~dB}\right)$ and reflection coefficients $\left(S_{11}>-0.7 \mathrm{~dB}\right)$. This for a control reverse voltage $U_{\text {com }}=V_{\mathrm{R}}=11.3 \mathrm{~V}$.

For a frequency $f=925 \mathrm{MHz}$, the transmission coefficient $S_{21}=-30 \mathrm{~dB}$ and the reflection coefficient $S_{11}=-0.4 \mathrm{~dB}$. These results show that the RF switch is really open.

\section{- Commutation time of the realized RF switch}

Figure 10 shows the output signal of the RF switch (Fig. 2) when controlled by a rectangular shaped voltage. The amplitude of this voltage switches between two levels $(7 \mathrm{~V}$ and $11.3 \mathrm{~V})$. This RF signal was measured by using a spectrum analyzer. From this signal we measured the switching times of this RF switch. The rise time $t_{\mathrm{ON}}=4 \mu \mathrm{s}$ and the descent time $t_{\mathrm{OFF}}=14 \mu \mathrm{s}$.

\section{Improving of the Performances of the RF Switch}

To improve the performance of the RF switch, we cascaded two resonant circuits. Figure 11 shows the new topology of the RF switch with two resonant circuits. 


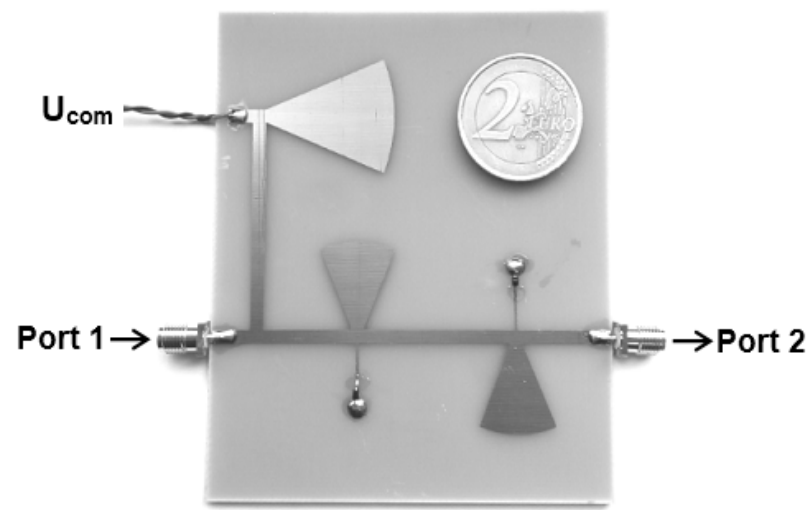

Fig. 11. New topology of the RF switch.

\subsection{Coefficient of Reflection of the BB149A Diode}

The simulation of the new topology of the RF switch, under the ADS software, requires knowledge of the reflection coefficient (module and phase) of the BB149A varicap diode. This can be seen through the two reverse control voltages $6.5 \mathrm{~V}$ and $12.2 \mathrm{~V}$. The $6.5 \mathrm{~V}$ control voltage ensures the closing of the RF switch (parallel resonance mode) and the $12.2 \mathrm{~V}$ control voltage ensures the opening of the RF switch (serial resonance mode). Figure 12 shows these reflection coefficients.

The results in Fig. 12 are recorded in Touchstone $\mathrm{s} 1 \mathrm{p}$ files. These describe the actual behavior of the varicap diode BB149A, when simulating the RF switch under the ADS software, when $V_{\mathrm{R}}=6.5 \mathrm{~V}$ or $V_{\mathrm{R}}=12.2 \mathrm{~V}$.

\subsection{Parameters $\mathrm{S}$ of the realized RF Switch}

The application of an inverted control inverse voltage equal to $6.5 \mathrm{~V}$ causes the closing of the RF switch. The resonant circuits of Fig. 11 operate in parallel resonance mode. They have infinite impedances and the RF switch is
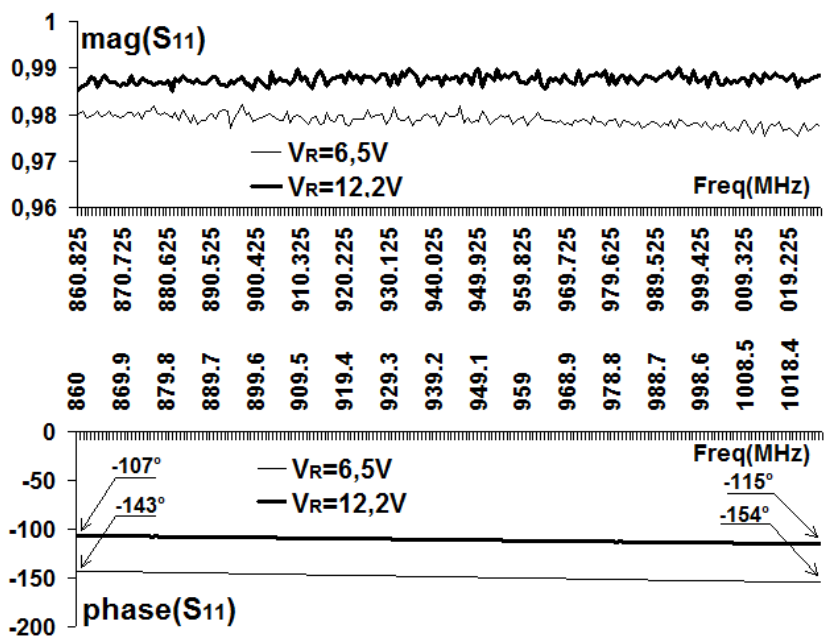

Fig. 12. Contents of two s1p files of varicap diode BB149A (for $V_{\mathrm{R}}=6.5 \mathrm{~V}$ or $V_{\mathrm{R}}=12.2 \mathrm{~V}$ ).
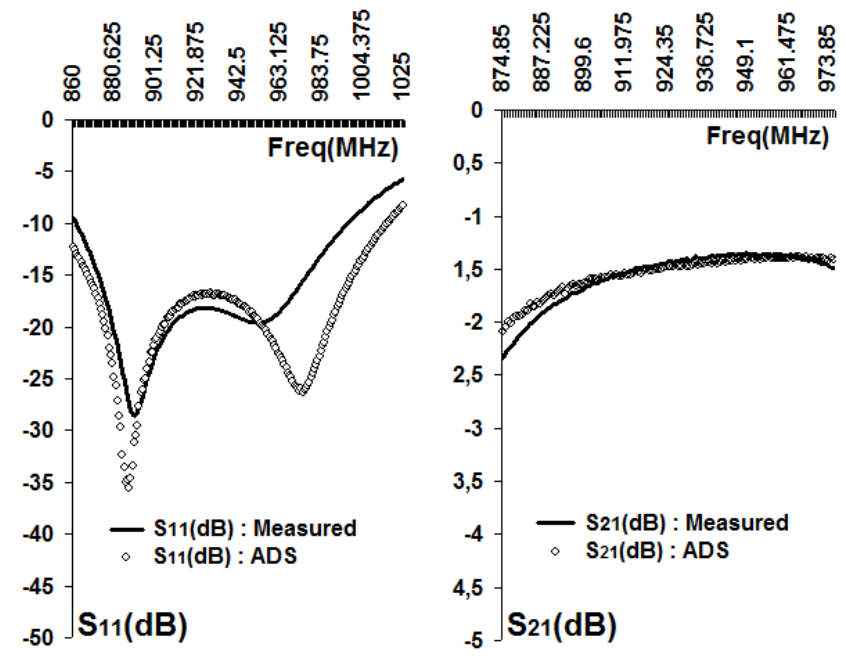

Fig. 13. Variation of $S_{11}$ and $S_{21}$ as a function of frequency $\left(V_{\mathrm{R}}=6.5 \mathrm{~V}\right)$.

transformed into a transmission line with characteristic impedance $Z_{\mathrm{C}}=50 \Omega$. This makes it possible to manage a considerable power, without distortion of the RF signal. The transmission between ports 1 and 2 of this switch is bidirectional with good linearity with respect to PIN diodes and FET transistors.

Figure 13 shows the variations of the transmission and reflection coefficients, when the RF switch is in the on-state $\left(S_{21}=-1.48 \mathrm{~dB}\right.$ and $S_{11}=-18.2 \mathrm{~dB}$ for $f=$ $925 \mathrm{MHz}$ and $V_{\mathrm{R}}=6.5 \mathrm{~V}$ ). The input and output of the RF switch are well adapted.

The application of a control reverse voltage equal to 12.2 V causes the RF switch to open. The resonant circuits of Fig. 11 operate in serial resonance mode. They have zero impedances, which short-circuits the transmission line and block the passage of the RF signal between ports 1 and 2 .

By a comparison in Fig. 9, Figure 14 clearly shows that the isolation of the RF switch was almost doubled in the off-state $\left(S_{21}=-53 \mathrm{~dB}\right.$ and $S_{11}=-0.4 \mathrm{~dB}$ for $f=$ $925 \mathrm{MHz}$ and $\left.V_{\mathrm{R}}=12.2 \mathrm{~V}\right)$.
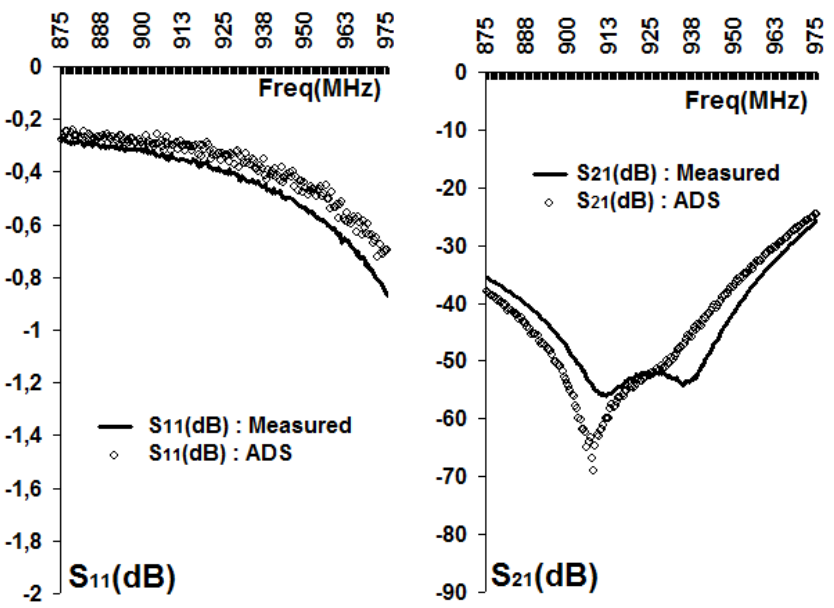

Fig. 14. Variation of $S_{11}$ and $S_{21}$ as a function of frequency $\left(V_{\mathrm{R}}=12.2 \mathrm{~V}\right)$. 
During the opening of our RF switch, we observe a total reflection (Fig. 14) due to the short circuit produced by the resonant circuit designed around the varicap diode. This influences the input and output impedance matching of our RF switch which brings us to the different applications, where the power is considerable, to provide an isolator RF to protect the RF source supplying power to our circuit.

We also deduce from the measurement result of the transmission coefficient (Fig. 14), that the two resonant circuits do not have the same resonance frequency $\left(f_{\mathrm{r} 1}=\right.$ $911 \mathrm{MHz}$ and $\mathrm{f}_{\mathrm{r} 2}=935 \mathrm{MHz}$ ). This is probably due either to the voltage drop between the two varicap diodes, the two resonant circuits $\left(V_{\mathrm{R} 1} \neq V_{\mathrm{R} 2} \Rightarrow C_{\mathrm{V} 1} \neq \mathrm{C}_{\mathrm{V} 2} \Rightarrow f_{\mathrm{r} 1} \neq f_{\mathrm{r} 2}\right.$ ), or to the two varicap diodes which may have a slight difference between values of the junction capacitances $\left(C_{\mathrm{V} 1} \neq C_{\mathrm{V} 2} \Rightarrow\right.$ $f_{\mathrm{r} 1} \neq f_{\mathrm{r} 2}$ ), or to a difference in the discontinuities of the two resonant circuits produced by the soldering points.

The presented switch is composed of resonating structure and is then inherently narrowband. It can be designed and tuned for any given application but in its present form it is not intended to cover wide bandwidths.

For some applications, the tuning voltage $\left(V_{\mathrm{R}}=6.5 \mathrm{~V}\right.$ or $V_{\mathrm{R}}=12.2 \mathrm{~V}$ ) is considered high enough or not available on board, this can be resolved either by selecting the appropriate varactor or by choosing the accurate values of inductors in the resonant structure in order to keep the voltage in the desired range.

\section{Conclusion}

In this article, we presented a new RF switch topology, made in microstrip technology. It has multiple advantages such as low manufacturing cost, simplicity of design, ease of reproducibility, low insertion loss, low power consumption and good linearity over PIN diodes and FET transistors. It is bi-directional and its control is based on a resonant circuit, placed in parallel with the load. The switching of the latter, of a series resonance (open switch) to a parallel resonance (closed switch) is provided by a varicap diode. The latter is a frequently available and inexpensive component. It is controlled by a DC voltage and operates with an almost negligible reverse bias current $(10 \mathrm{nA})$, which is much lower than that required by a PIN diode $(10 \mathrm{~mA})$. This switch has been tested and characterized in the UHF band. Its switching time is of the order of microseconds. In the off-state, it has good insulation, of the order of $50 \mathrm{~dB}$. In the on-state, the resonant circuit has an infinite impedance and the RF switch is transformed into a transmission line with a characteristic impedance $\mathrm{Z}_{\mathrm{C}}=50 \Omega$. This makes it possible to manage a considerable power, without distortion of the RF signal and with a low insertion loss. The size of the realized RF switch can be considerably reduced by replacing the distributed elements (inductances and capacitors) with localized elements.

\section{Acknowledgments}

This work has been supported and funded by the Sys'Com Research Laboratory, National Engineering School of Tunis (ENIT) and University Tunis El Manar, Tunis, Tunisia.

\section{References}

[1] ALAM, M. S., ABBOSH, A. M. Beam-steerable planar antenna using circular disc and four pin-controlled tapered stubs for WiMAX and WLAN applications. IEEE Antennas and Wireless Propagation Letters, 2016, vol. 15, p. 980-983. DOI: 10.1109/LAWP.2015.2489684

[2] LU, P., YANG, X. S., LI, J. L., et al. Polarization reconfigurable broadband rectenna with tunable matching network for microwave power transmission. IEEE Transactions on Antennas and Propagation, 2016, vol. 64, no. 3, p. 1136-1141. DOI: 10.1109/TAP.2016.2518198

[3] YANG, X. L., LIN, J. C., CHEN, G., et al. Frequency reconfigurable antenna for wireless communications using $\mathrm{GaAs}$ FET switch. IEEE Antennas and Wireless Propagation Letters, 2015, vol. 14, p. 807-810. DOI: 10.1109/LAWP.2014.2380436

[4] NGUYEN, D. P., PHAM, A. V., ARYANFAR, F. A k-band high power and high isolation stacked-FET single pole double throw MMIC switch using resonating capacitor. IEEE Microwave and Wireless Components Letters, 2016, vol. 26, no. 9, p. 696-698. DOI: 10.1109/LMWC.2016.2597235

[5] RAJAGOPALAN, H., KOVITZ, J. M., RAHMAT-SAMII, Y. MEMS reconfigurable optimized e-shaped patch antenna design for cognitive radio. IEEE Transactions on Antennas and Propagation, 2014, vol. 62, no. 3, p. 1056-1064. DOI: 10.1109/TAP.2013.2292531

[6] ANAGNOSTOU, D. E., CHRYSSOMALLIS, M. T., BRAATEN, B. D., et al. Reconfigurable UWB antenna with RF-MEMS for ondemand WLAN rejection. IEEE Transactions on Antennas and Propagation, 2014, vol. 62, no. 2, p. 602-608. DOI: 10.1109/TAP.2013.2293145

[7] RICHARDS, R. J., DE LOS SANTOS, H. J. MEMS for RF/Microwave Wireless Applications: The Next Wave. Tutorial. 2001. [Online] Available at: https://www.researchgate.net/profile/Hector_De Los Santos3/pub lication/237726374_MEMS_for_RFmicrowave_wireless_applicati ons The next wave/links/54 d27 c870cf2b0c61469be98/MEMSfor-RF-microwave-wireless-applications-The-next-wave.pdf

[8] Varicap diode, https://en.wikipedia.org/wiki/Varicap

[9] KALAMKAR, S. Design and analysis of frequency reconfigurable microstrip patch antenna. International Journal of Innovative Research in Computer and Communication Engineering (IJIRCCE), 2017, vol. 5, no. 3, p. 4390-4395. DOI: 10.15680/IJIRCCE.2017. 0503144

[10] HINSZ, L., BRAATEN, B. D. A frequency reconfigurable transmitter antenna with autonomous switching capabilities. IEEE Transactions on Antennas and Propagation, 2014, vol. 62, no. 7, p. 3809-3813. DOI: 10.1109/TAP.2014.2316298

[11] Microstrip, https://en.wikipedia.org/wiki/Microstrip

[12] WADELL, B. C. Transmission Line Design Handbook. Norwood (MA, USA): Artech House, 1991 (p. 300). ISBN: 0-89006-436-9 
[13] HONG J. S., LANCASTER, M. J. Microstrip Filters for RF/Microwave Applications. John Wiley \& Sons, 2001. (p. 90, p. 97-99, p. 188-189) ISBN: 0-471-22161-9

[14] FOOKS, E. H., ZAKAREVICIUS, R. A. Microwave Engineering using Microstrip Circuits. Prentice Hall of Australia, 1990. (p. 13, p. 93, p. 102, p. 104) ISBN: 0136916503

[15] BAHL, I., BHARTIA, P. Microwave Solid State Circuit Design. $2^{\text {nd }}$ ed. Hoboken (NJ, USA): John Wiley \& Sons, 2003. (p. 290) ISBN: 0-471-20755-1

[16] PHILIPS. BB149A UHF Variable Capacitance Diode. Product data sheet rev. 03 (2004).

[17] Touchstone File Format Specification. https: //ibis.org/connector/touchstone_spec11.pdf

\section{About the Authors ...}

Fethi MEJRI was born in Tunisia. He received the diploma of engineer in Electronics from the National Engineering School of Tunis (ENIT), in 1993, the Diploma of
Advanced Study in Analysis of Digital Systems and Processing from the ENIT, in 1998, and the doctoral degree in Telecommunications from the ENIT, in 2006. Now he is with the Faculty of Science of Bizerte (FSB), Tunisia, as an Assistant Professor. His research activities include electromagnetic modeling, design and realization of microwave circuits.

Taoufik AGUILI was born in Tunisia. He received the diploma of DEA in Integrated Electronics from INSA Lyon, in 1987, the Diploma doctorate in Integrated Electronics from UCBL Lyon, in 1990, and the Diploma of doctorate of state in Electrical Engineering of the National School of Engineers of Tunis, in 1999. He was appointed the Head of "Communications Systems" at the ENIT, in 1998, a Professor in Telecommunications at the National School of Engineers of Tunis, in 2004, and the Director of Communications Systems Laboratory at ENIT, in 2011. His research activities include electromagnetic microwave circuits modeling and analysis of scattering and propagation phenomena in free space. 